\title{
Electrostatic equilibria of non-neutral plasmas confined in a Penning trap with axially varying magnetic field
}

\author{
Cite as: Phys. Plasmas 26, 052511 (2019); doi: 10.1063/1.5092136 \\ Submitted: 7 February 2019 - Accepted: 30 April 2019 • \\ Published Online: 21 May 2019
}
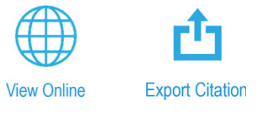

R. A. Lane ${ }^{1,2}$ and C. A. Ordonez ${ }^{1, a)}$ (iD

\author{
AFFILIATIONS \\ ${ }^{7}$ Department of Physics, University of North Texas, Denton, Texas 76203, USA \\ ${ }^{2}$ Ball Aerospace, Albuquerque, New Mexico 87106, USA
}

a) Author to whom correspondence should be addressed: cao@unt.edu

\begin{abstract}
A procedure for computing the electrostatic equilibria of non-neutral plasmas in a Penning trap with a nonuniform magnetic field by solving Poisson's equation using an iterative method is described. Plasma equilibria in a model Penning trap with high and low field regions are computed. The plasma is assumed to follow the Boltzmann density distribution along magnetic field lines. Correspondence with previous investigations examining similar configurations analytically and using particle-in-cell simulations is found. The relationship between the plasma density in low and high field regions is examined for various plasma temperatures, densities, magnetic mirror ratios, and plasma and electrode radii. An analytical description of the radial density profile in the high field region is developed and compared with the computed equilibria. A concept is described for cooling a positron plasma with laser-cooled ions trapped axially within a high magnetic field region, while antiprotons are trapped axially separated from the laser-cooled ions within a low field region, and the positron plasma extends to both regions.
\end{abstract}

Published under license by AIP Publishing. https://doi.org/10.1063/1.5092136

\section{INTRODUCTION}

Equilibria of non-neutral plasmas are evaluated in a model Penning trap with an axially varying magnetic field, in the work presented here. Simulated equilibria are considered to follow the Boltzmann density distribution along magnetic field lines. Equilibria are calculated by solving Poisson's equation self-consistently using a finite-difference computational approach, ${ }^{1}$ with the approach adapted for the axially varying magnetic field. Electrostatic potential profiles of non-neutral plasmas containing oppositely charged species have been previously computed using similar methods in multiple trap geometries with uniform magnetic fields ${ }^{2,3}$ and no magnetic field. ${ }^{4}$ The effects of incorporating magnetic mirrors on non-neutral plasma confinement in Penning traps have been studied analytically, ${ }^{5}$ using particle-in-cell (PIC) simulations, ${ }^{6,7}$ and experimentally. ${ }^{5,7}$ The results from the current study show correspondence with the previous analytical and PIC results.

The work presented here is an extension and generalization of previous work, which predicted that electrostatic, space charge based antiproton confinement is possible specifically within an existing Penning trap based antihydrogen experiment. ${ }^{8}$ In the present work, the relationship between the plasma density in low and high field regions is examined for various plasma temperatures, densities, magnetic mirror ratios, and plasma and electrode radii. An analytical description of the axial density variation is developed following a procedure similar to previous analytical studies. ${ }^{5}$ The analytical description is compared with self-consistently computed equilibria.

The possibility of using laser-cooled ions to sympathetically cool antimatter plasmas for antihydrogen production has been investigated. ${ }^{9}$ Here, a possible configuration is described for a three-species plasma confined in a Penning trap with axially varying magnetic fields. In the configuration, the space charge of a positron plasma generates a potential difference along the central axis. The potential difference is used to keep laser-cooled ions axially separated from antiprotons, while both species interact with the positron plasma.

In Ref. 4, a theoretical understanding was developed of the conditions for unmagnetized plasma ions (or positrons) to be confined by the space charge of an electron plasma. The electron plasma would have a nearly full Maxwellian velocity distribution at the plasma edge. Only electrons in the tail of the distribution have enough energy to travel from the plasma edge to the geometric center of the 
unmagnetized bulk plasma, which consists of both ions and electrons. It may seem to be counterintuitive, but it was found that, in a selfconsistently relaxed equilibrium, the magnitude of the difference in electric potential energy of an electron or singly charged ions between the geometric center of the bulk plasma and the plasma edge can be large compared to the electron and ion temperatures in energy units, provided that the electron density at the edge is much larger than that at the center. ${ }^{4}$ Consequently, near-perfect electrostatic confinement of plasma ions using the space charge of a nondrifting electron plasma, while each species is sufficiently relaxed to follow a Boltzmann density distribution, is predicted to be possible. In Ref. 4, the exact structure of the reflecting boundary used to confine an electron plasma along its edge was not considered. The possibility of using a sequence of magnetic cusps with the addition of electrostatic plugging is described in Ref. 10, where a classical trajectory Monte Carlo study of single particle trajectories was reported. The present work contributes to an improved theoretical understanding of how a non-neutral electron plasma responds self-consistently to a spatially changing magnetic field, such as within a magnetic cusp or Penning trap with an axially varying magnetic field.

The details of the model trap with an axially varying magnetic field are given in Sec. II. The computational methods are described in Sec. III. Equilibria are presented in Sec. IV, and an analytical description of the equilibria is developed using normalized co-ordinates in Sec. V. A possible application is described in Sec. VI, and a discussion is provided in Sec. VII.

\section{MODEL}

Equilibria of a non-neutral plasma in a model Penning trap with an axially varying magnetic field are computed. The model and its magnetic field are cylindrically symmetric and are illustrated in Fig. 1. The model has a continuous magnetic field that transitions between high and low field strength regions. The high and low field regions have magnetic field strengths $B_{H}$ and $B_{L}$, respectively.

The model magnetic field is generated by two solenoids that are coaxial with the $z$ axis. One solenoid is considered to be long enough to produce a uniform field, $\mathbf{B}_{L}=B_{L} \hat{\mathbf{z}}$, throughout the region of interest. A smaller, finite-length solenoid extends from $z=-L_{S}$ to $z=0$ with radius $r_{S}$. The combined magnetic field at a reference point inside the finite-length solenoid $\left(0,-z_{0}\right)$, is $\mathbf{B}_{H}=B_{H} \hat{\mathbf{z}}$. Dimensions are chosen such that $L_{S} \gg z_{0} \gg r_{S}$. The magnetic field of a finite-length solenoid is given in the Appendix. The magnitude $B_{H}$ can be expressed as a multiple of $B_{L}, B_{H}=R B_{L}$, where $R$ is the mirror ratio.

The model is radially bound by a grounded, cylindrical electrode that is coaxial with the solenoids. The cylindrical electrode has inner radius $r_{W}$, where $r_{W}<r_{S}$. The cylindrical electrode introduces a Dirichlet boundary condition to the potential at $r=r_{W}$, and cylindrical symmetry gives a Neumann boundary condition at $r=0$. The trap is assumed to be much longer than the computational region, and Neumann boundary conditions are applied at $\pm z_{0}$ to simulate a system that continues axially to $|z| \gg z_{0}$. Symbolically, the boundary conditions on the electrostatic potential $\phi$ are

$$
\begin{gathered}
\phi\left(r_{W}, z\right)=0, \\
\left.\frac{\partial}{\partial r} \phi(r, z)\right|_{r=0}=0,
\end{gathered}
$$
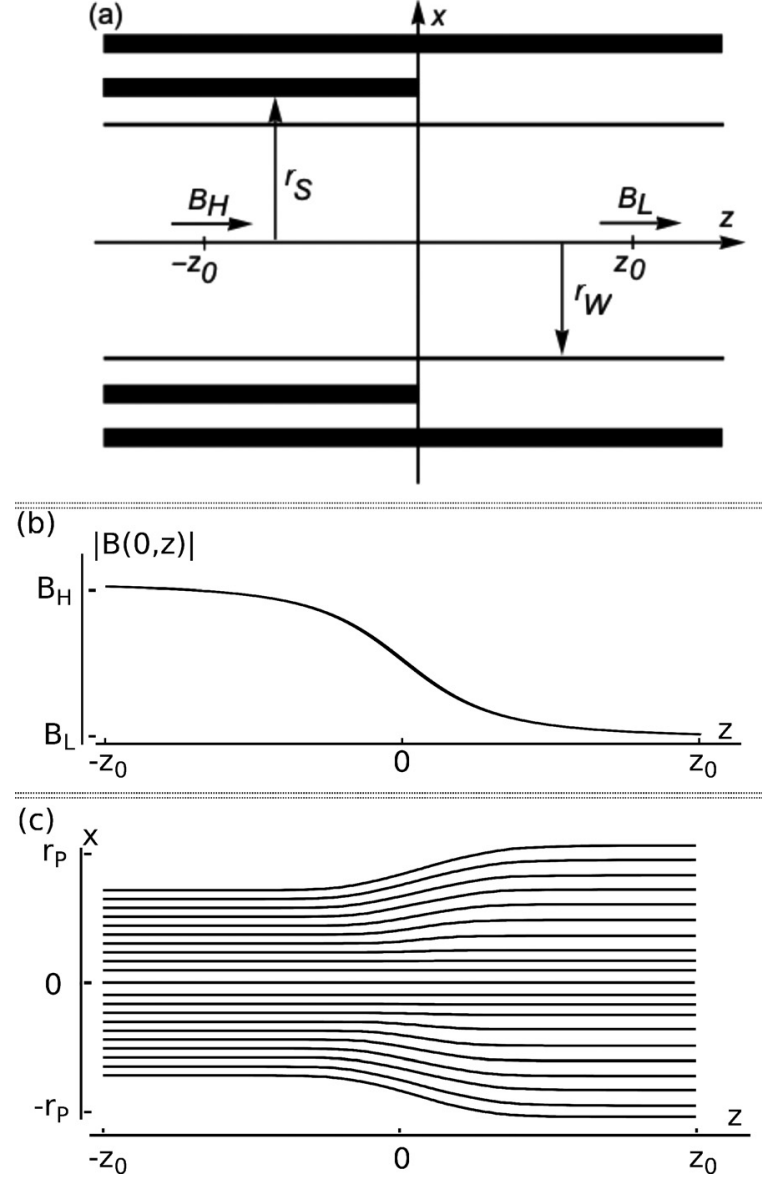

FIG. 1. The model consists of two solenoids coaxial with a grounded cylindrical electrode and is sketched in (a). The solenoids generate a magnetic field that varies axially from a high field $B_{H}$ at $z=-z_{0}$ to a low field $B_{L}$ at $z=z_{0}$. The magnetic field along the $z$ axis is plotted in (b) and magnetic field lines that progress from left to right are shown in (c).

$$
\left.\frac{\partial}{\partial z} \phi(r, z)\right|_{z= \pm z_{0}}=0 \text {. }
$$

As indicated above, the system considered here is cylindrically symmetric. The co-ordinates $(r, z)$ represent a point in three-dimensional space specified by cylindrical co-ordinates, with the azimuthal angle co-ordinate omitted for brevity.

\section{COMPUTATIONS}

Equilibria are computed by solving Poisson's equation, $\nabla^{2} \phi(\mathbf{r})$ $=-e n(\mathbf{r}) / \epsilon_{0}$, through a self-consistent finite-difference computational approach, ${ }^{1}$ with the approach adapted for the axially varying magnetic field. The model non-neutral plasma is composed of positrons with density $n(\mathbf{r})$, each positron has charge $e$, and the vacuum permittivity is $\epsilon_{0}$.

The positron radial density profile is specified at $z=z_{0}$. The radial positron distribution in the $z=z_{0}$ plane is uniform out to a radius $r=r_{P}$, where it possesses a sharp edge. Symbolically, the density is $n\left(r, z_{0}\right)=n_{0} \Theta\left(r_{P}-r\right)$, where $n_{0}$ is the positron density at $z=z_{0}$, and 
$\Theta$ is the Heaviside step function defined as $\Theta(x<0)=0$, $\Theta(x \geq 0)=1$. If the positron plasma follows the Boltzmann density relation along curved magnetic field lines, the density at any point is

$$
n(r, z)=n_{0} \Theta\left(r_{P}-r_{0}\right) \exp \left(-\frac{e}{k_{B} T}\left[\phi(r, z)-\phi\left(r_{0}, z_{0}\right)\right]\right) .
$$

Here, $T$ is the positron plasma temperature, $k_{B}$ is Boltzmann's constant, and $r_{0}$ is the radial position where a magnetic field line passing through $(r, z)$ intersects the $z=z_{0}$ plane. Magnetic field lines are solutions to $\mathrm{d} r / B_{r}(r, z)=\mathrm{d} z / B_{z}(r, z)$, which is solved in parametric form

$$
\begin{aligned}
& \frac{\mathrm{d} r}{\mathrm{~d} s}=\frac{B_{r}(r(s), z(s))}{|\mathbf{B}(r(s), z(s))|}, \\
& \frac{\mathrm{d} z}{\mathrm{~d} s}=\frac{B_{z}(r(s), z(s))}{|\mathbf{B}(r(s), z(s))|} .
\end{aligned}
$$

The electrostatic potential is computed in the region $0 \leq r \leq r_{W}$ and $-z_{0} \leq z \leq z_{0}$. A uniform computational grid with axial spacing $\Delta z$ and radial spacing $\Delta r$ is used. Grid spacing is chosen to be less than $\lambda_{D} /(2 R)$, where $\lambda_{D}=\left[\epsilon_{0} k_{B} T /\left(e^{2} n_{0}\right)\right]^{1 / 2}$ is the Debye length. Generally, mesh spacing of less than $\lambda_{D} / 2$ is needed to ensure convergence. ${ }^{1}$ Here, a factor of the mirror ratio $R$ is included to accommodate increased density in the high field region.

Radial grid points take values $n \Delta r$, where $n$ is an integer. The position $r_{0}$ is calculated for each grid point through Eq. (3) using a fourth-order Runge-Kutta solver. Values of $r_{0}$ fall between grid points such that $n \Delta r<r_{0}<(n+1) \Delta r$. The potential $\phi\left(r_{0}, z_{0}\right)$ used in Eq. (2) is given by the weighted average

$$
\begin{aligned}
\phi\left(r_{0}, z_{0}\right)= & \left(1-\frac{r_{0}-n \Delta r}{\Delta r}\right) \phi\left(n \Delta r, z_{0}\right) \\
& +\frac{r_{0}-n \Delta r}{\Delta r} \phi\left((n+1) \Delta r, z_{0}\right) .
\end{aligned}
$$

A finite-difference method is used with central-differencing to find self-consistent solutions to Poisson's equation. ${ }^{1}$ Solutions are found by relaxation from an arbitrary initial solution $\phi^{(0)}(r, z)$. Subsequent iterations are obtained by finding the solution at each grid point through

$$
\begin{aligned}
\phi^{(l+1)}(r, z)= & -(\omega-1) \phi^{(l)}(r, z)+\frac{\omega}{2}\left(\frac{1}{\Delta r^{2}}+\frac{1}{\Delta z^{2}}\right)^{-1} \\
& \times\left[\frac{\phi^{(l)}(r+\Delta r, z)-\phi^{(l)}(r-\Delta r, z)}{2 r \Delta r}\right. \\
& +\frac{\phi^{(l)}(r+\Delta r, z)+\phi^{(l)}(r-\Delta r, z)}{\Delta r^{2}} \\
& \left.+\frac{\phi^{(l)}(r, z+\Delta z)+\phi^{(l)}(r, z-\Delta z)}{\Delta r^{2}}+\frac{e}{\epsilon_{0}} n^{(l)}(r, z)\right]
\end{aligned}
$$

where $\omega$ is a mixing constant, and iterations are continued until the solution converges to within a selected tolerance.

\section{EQUILIBRIA}

A "base case" is chosen where the model dimensions and plasma parameters approximately correspond to a particle-in-cell study of Penning traps with magnetic mirrors. ${ }^{6}$ The positron plasma has parameters $n_{0}=1.0 \times 10^{13} \mathrm{~m}^{-3}, T=1000 \mathrm{~K}$, and $r_{P}=5 \mathrm{~mm}$. The electrode inner radius is $r_{W}=20 \mathrm{~mm}$, and the finite-length solenoid has an inner radius of $r_{S}=30 \mathrm{~mm}$ with a length of $L_{S}=3.0 \mathrm{~m}$. The current $I_{S}$ and number of windings per unit length $N_{S}$ are chosen to produce a magnetic field of $1.0 \mathrm{~T}$ at $\left(r=0, z=-z_{0}=-15 \mathrm{~cm}\right)$ for a total field of $B_{H}=2.0 \mathrm{~T}$ or $R=2.0$ when superimposed with the uniform field $B_{L}=1.0 \mathrm{~T}$.

An electrostatic potential difference along the $z$ axis is found to form self-consistently. The maximum potential, the axial potential difference, the ratio of the density at $\left(0,-z_{0}\right)$ to $n_{0}$, and the full width at half maximum density (FWHM) of the positron plasma are computed for each equilibrium. The maximum potential is $\phi_{\max }=\phi\left(0, z_{0}\right)$, and the axial potential difference is given by $\Delta \phi=\phi_{\max }-\phi\left(0,-z_{0}\right)$. The ratio of the density at $\left(0,-z_{0}\right)$ to the density $n_{0}$ is referred to as the density ratio, and the FWHM for the positron plasma is given at $z=-z_{0}$. The density $n_{0}$ is the density at $\left(r \leq r_{p}, z=z_{0}\right)$.

The electrostatic potential of the base case is shown in Fig. 2, and the positron density is shown in Fig. 3. In the base case, the maximum potential is $\phi_{\max }=4.2 \mathrm{~V}$, and the axial potential difference is $\Delta \phi=57 \mathrm{mV}$. The density ratio is 1.94 , and the FWHM of the positron plasma at $z=-z_{0}=-15 \mathrm{~cm}$ is $6.9 \mathrm{~mm}$. In comparison, the width of the positron plasma at $z=z_{0}=15 \mathrm{~cm}$ is $2 r_{P}=10 \mathrm{~mm}$. The field line that crosses the $z=z_{0}$ plane at $r=r_{P}=5 \mathrm{~mm}$ crosses the $z=-z_{0}$ plane at $r=3.65 \mathrm{~mm}$.

The relationships between the density, temperature, plasma radius, magnetic mirror ratio, and electrode radius are investigated by equilibria computations under individual changes of these parameters. The maximum potential, the axial potential difference, the density ratio, the FWHM at $z=-z_{0}$, and the normalized parameter $\Delta \psi=e \Delta \phi /\left(k_{B} T\right)$ are reported for each equilibrium in Table I. The base case values are listed at the top of Table I. The parameter that is varied from the base case is listed in the first column, and its value is listed in the second.

For the parameter space considered, the maximum potential does not vary by more than $2 \%$ under changes in temperature of up to two orders of magnitude (from $T=500 \mathrm{~K}$ to $T=5 \times 10^{4} \mathrm{~K}$ ) or changes to the magnetic mirror ratio of up to a factor of 7.3 (from $R=1.5$ to $R=11$ ). In the same range of temperature variations, the axial potential difference changes by up to a factor of 37 (from

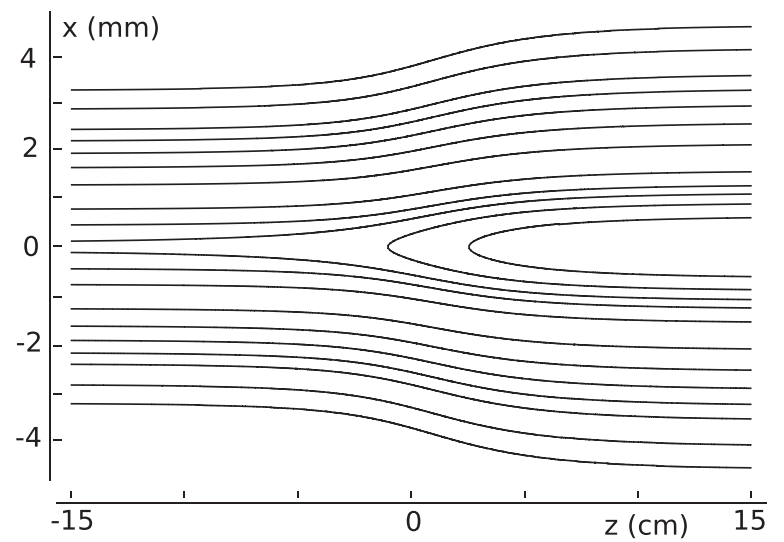

FIG. 2. The computed electrostatic potential of the base case is shown as a contour plot. 


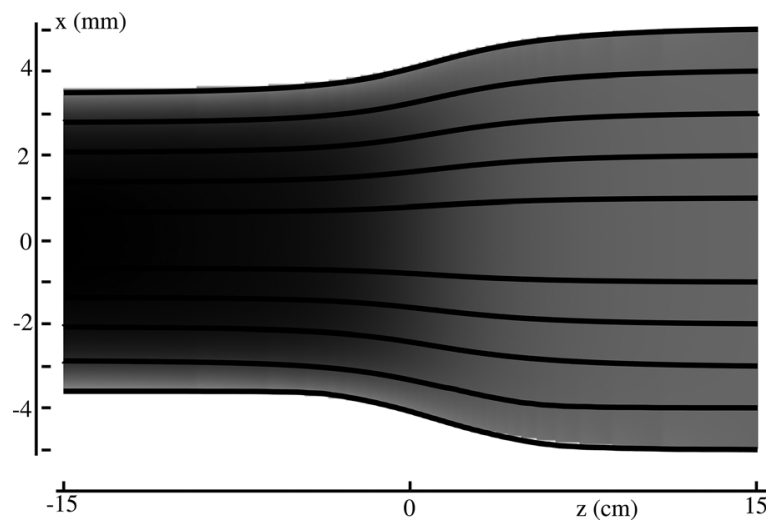

FIG. 3. The equilibrium positron density for the base case is shown with darker hues representing regions of higher density. Magnetic field lines are shown as black lines.

$28.8 \mathrm{mV}$ to $1070 \mathrm{mV}$ ). The range of changes to the magnetic mirror ratio produces changes in the axial potential difference of up to a factor of 5.9 (from $33.4 \mathrm{mV}$ to $198 \mathrm{mV}$ ). The density variations induce changes to the axial potential difference by a maximum of $25 \%$ of the base case value.

\section{ANALYTICAL DESCRIPTION}

In Ref. 5, an expression for the potential difference along a magnetic field line in finite-temperature flat-top plasmas with high and

TABLE I. Equilibria are found for parameter sets with one parameter varied from the base case, for which $n_{0}=1.0 \times 10^{13} \mathrm{~m}^{-3}, T=1000 \mathrm{~K}, r_{P}=5 \mathrm{~mm}, R=2.0$, and $r_{W}=20 \mathrm{~mm}$. The parameter varied is given in the first column, and the parameter value is given in the second. The maximum potential $\phi_{\max }$, axial potential difference $\Delta \phi$, ratio of the density at $\left(0,-z_{0}\right)$ to $n_{0}$, the full width at half maximum density (FWHM) of the positron plasma at $z=-z_{0}$, and the normalized parameter $\Delta \psi$ $=e \Delta \phi /\left(k_{B} T\right)$ are given. The base case equilibrium values are reported at the top of the table.

\begin{tabular}{|c|c|c|c|c|c|c|}
\hline \multicolumn{2}{|c|}{ Parameter } & \multirow{2}{*}{$\begin{array}{c}\phi_{\max } \\
(\mathrm{V})\end{array}$} & \multirow{2}{*}{$\begin{array}{c}\Delta \phi \\
(\mathrm{mV})\end{array}$} & \multirow{2}{*}{$\begin{array}{c}n\left(0,-z_{0}\right) / n_{0} \\
\quad \text { (Unitless) }\end{array}$} & \multirow{2}{*}{$\begin{array}{c}\text { FWHM } \\
(\mathrm{mm})\end{array}$} & \multirow{2}{*}{$\begin{array}{c}\Delta \psi \\
\text { (Unitless) }\end{array}$} \\
\hline Symbol & Value & & & & & \\
\hline Base & & 4.22 & 57.2 & 1.94 & 6.9 & 0.664 \\
\hline$n_{0}$ & $1.0 \times 10^{12} \mathrm{~m}^{-3}$ & 0.422 & 42.9 & 1.65 & 7.3 & 0.498 \\
\hline$n_{0}$ & $5.0 \times 10^{12} \mathrm{~m}^{-3}$ & 2.11 & 55.6 & 1.91 & 7.3 & 0.645 \\
\hline$r_{P}$ & $2.5 \mathrm{~mm}$ & 1.43 & 53.2 & 1.85 & 3.7 & 0.617 \\
\hline$r_{P}$ & $10 \mathrm{~mm}$ & 10.67 & 57.7 & 1.95 & 12.0 & 0.670 \\
\hline$T$ & $500 \mathrm{~K}$ & 4.22 & 28.8 & 1.95 & 6.7 & 0.668 \\
\hline$T$ & $5000 \mathrm{~K}$ & 4.22 & 249 & 1.78 & 7.3 & 0.578 \\
\hline$T$ & $10^{4} \mathrm{~K}$ & 4.23 & 429 & 1.65 & 7.3 & 0.498 \\
\hline$T$ & $5 \times 10^{4} \mathrm{~K}$ & 4.23 & 1070 & 1.28 & 7.3 & 0.248 \\
\hline$R$ & 1.5 & 4.22 & 33.4 & 1.47 & 8.4 & 0.388 \\
\hline$R$ & 4.0 & 4.20 & 115 & 3.79 & 4.4 & 1.33 \\
\hline$R$ & 5.0 & 4.19 & 134 & 4.69 & 3.8 & 1.56 \\
\hline$R$ & 11 & 4.14 & 198 & 9.85 & 2.4 & 2.30 \\
\hline$r_{W}$ & $10 \mathrm{~mm}$ & 2.67 & 56.8 & 1.93 & 6.3 & 0.659 \\
\hline$r_{W}$ & $40 \mathrm{~mm}$ & 5.47 & 57.1 & 1.94 & 9.1 & 0.663 \\
\hline
\end{tabular}

low magnetic field regions is derived for magnetic mirror ratios with $R=1+\beta$ and $\beta \ll 1$. The axial potential difference $\Delta \phi$ is estimated for the equilibria in Table I using the expression from Ref. 5. For the base case equilibrium with $R=2.0$, the analytical expression from Ref. 5 overestimates $\Delta \phi$ by approximately a factor of 1.5 , while for the equilibrium with $R=1.5$, the analytical expression is within $30 \%$ of the computed value. The expression overestimates the computed value for equilibria with $R>2.0$ by a larger factor of up to approximately 4 when $R=11$ and other parameter values are those of the base case.

An expression for the radial density profile in the high field region, $n\left(r,-z_{0}\right)$, is derived here, using a similar approach to Ref. 5 . The derivation assumes that the density distribution in the low field region, $n\left(r, z_{0}\right)$, is known. A normalized expression for $n\left(r,-z_{0}\right)$ is derived that gives the density variation's dependence on the magnetic mirror ratio, by considering a flat-top density profile at $z=z_{0}$.

A normalized co-ordinate system is applied to reduce the number of parameters involved in the description of the system. The normalized co-ordinate system allows the Poisson-Boltzmann governing equation applied here to be expressed in terms of the normalized potential $\psi=e\left(k_{B} T\right)^{-1} \phi$ as

$$
\nabla^{2} \psi(\rho, \zeta)=-\Theta\left(\rho_{P}-\rho_{0}\right) \exp \left[\psi\left(\rho_{0}, \zeta_{0}\right)-\psi(\rho, \zeta)\right] .
$$

The normalized co-ordinates are $\rho=r / \lambda_{D}$ and $\zeta=z / \lambda_{D}$, which dictate the form of normalized dimensions, $\rho_{W}=r_{W} / \lambda_{D}, \rho_{P}=r_{P} / \lambda_{D}, \rho_{0}=r_{0} /$ $\lambda_{D}$, and $\zeta_{0}=z_{0} / \lambda_{D}$. Here, $r_{W}$ is the electrode inner radius, $r_{P}$ is the plasma radius at $z=z_{0}$, and $r_{0}$ is defined as the radial co-ordinate at $z=z_{0}$ such that the same magnetic field line passes through the two points $\left(r_{0}, z_{0}\right)$ and $(r, z)$. The reduced set of parameters consists of the normalized dimensions $\rho_{P}, \rho_{W}$, and $\zeta_{0}$. In addition, the functional dependence, $\rho_{0}(\rho, \zeta)$, accounts for the magnetic field being nonuniform. With the boundary conditions specified by Eq. (1), only the boundary value $\psi\left(\rho_{W}, \zeta\right)$ needs to be specified. The electric potential is defined to within an arbitrary additive constant, and $\psi\left(\rho_{W}, \zeta\right)=0$ is used.

To develop an analytical description of the plasma at $z=-z_{0}$, the value of $z_{0}$ is considered to be sufficiently large for the magnetic field to be approximately uniform at both $z_{0}$ and $-z_{0}$. In such a limit, the analytical description does not depend on the value of $\zeta_{0}$. Nevertheless, the theory is developed referring to the axial locations $z=z_{0}$ and $z=-z_{0}$ for convenience.

The system is treated using the infinite-cylindrical-column approximation both in the vicinity of $z_{0}$, which is the low field region, and in the vicinity of $-z_{0}$, which is the high field region. The same magnetic field line is considered to pass through the two points $\left(r_{0}, z_{0}\right)$ and $\left(r,-z_{0}\right)$. If the azimuthal angle co-ordinate (which is not written for brevity) is given a continuous distribution of values between 0 and $2 \pi,\left(r_{0}, z_{0}\right)$ and $\left(r,-z_{0}\right)$ represent two circles. The same magnetic flux must pass through each circle (neglecting the plasma diamagnetic effect). Consequently, $B_{L} \pi r_{0}^{2}=B_{H} \pi r^{2}$, or, equivalently, $\rho_{0}=\sqrt{R} \rho$, where $R$ is the mirror ratio.

The radial electric potential profile at $z=z_{0}$ is approximated as that of a long cylindrical plasma with uniform density. The electric field inside the plasma is found from Gauss's law and is used to write an expression for the scalar potential

$$
\phi\left(r_{0}, z_{0}\right)=\frac{e}{\epsilon_{0}}\left[\ln \left(\frac{r_{W}}{r_{P}}\right) \int_{0}^{r_{P}} \mathrm{~d} r r n\left(r, z_{0}\right)+\int_{r_{0}}^{r_{P}} \frac{\mathrm{d} r^{\prime}}{r^{\prime}} \int_{0}^{r^{\prime}} \mathrm{d} r^{\prime \prime} r^{\prime \prime} n\left(r^{\prime \prime}, z_{0}\right)\right],
$$


where $r_{0}$ is the radial cylindrical co-ordinate at the axial location $z=z_{0}$. Equation (7) applies when $r_{0} \leq r_{P}$ and when the electric potential at the wall is defined to be zero, $\phi\left(r_{W}, z_{0}\right)=0$. A flat-top density profile, $n\left(r_{0}, z_{0}\right)=n_{0} \Theta\left(r_{P}-r_{0}\right)$, is used and allows an analytical solution to be found. Carrying out the integration and normalizing yields

$$
\psi\left(\rho_{0}, \zeta_{0}\right)=\frac{\rho_{P}^{2}}{2}\left[\ln \left(\frac{\rho_{W}}{\rho_{P}}\right)+\frac{1}{2}\right]-\frac{\rho_{0}^{2}}{4} .
$$

The radial electric potential profile at $z=-z_{0}$ is also approximated as that of a long cylindrical plasma, but with a density that is axially uniform and not radially uniform. Equation (7) applies at $z=-z_{0}$, provided that the replacements $z_{0} \rightarrow-z_{0}, r_{0} \rightarrow r$, and $r_{P}$ $\rightarrow r_{P H}$ are used, where the same magnetic field line passes through the two points $\left(r_{0}, z_{0}\right)$ and $\left(r,-z_{0}\right)$ and the same magnetic field line passes through the two points $\left(r_{P}, z_{0}\right)$ and $\left(r_{P H},-z_{0}\right)$. The resulting expression is

$$
\begin{aligned}
\phi\left(r,-z_{0}\right)= & \frac{e}{\epsilon_{0}}\left[\ln \left(\frac{r_{W}}{r_{P H}}\right) \int_{0}^{r_{P H}} \mathrm{~d} r r n\left(r,-z_{0}\right)\right. \\
& \left.+\int_{r}^{r_{P H}} \frac{\mathrm{d} r^{\prime}}{r^{\prime}} \int_{0}^{r^{\prime}} \mathrm{d} r^{\prime \prime} r^{\prime \prime} n\left(r^{\prime \prime},-z_{0}\right)\right],
\end{aligned}
$$

where $r$ is the radial cylindrical co-ordinate at the axial location $z=-z_{0}$. Let $f$ denote a normalized function to be found that gives the radial dependence of the density at $z=-z_{0}$. Defining $f$ such that $n\left(r,-z_{0}\right)=f\left(r / \lambda_{D}\right) n_{0} \Theta\left(r_{P H}-r\right)$, the normalized radial potential profile at $z=-z_{0}$ in the high field region is given by

$$
\psi\left(\rho,-\zeta_{0}\right)=\ln \left(\frac{\rho_{W}}{\rho_{P H}}\right) \int_{0}^{\rho_{P H}} \mathrm{~d} \rho \rho f(\rho)+\int_{\rho}^{\rho_{P H}} \frac{\mathrm{d} \rho^{\prime}}{\rho^{\prime}} \int_{0}^{\rho^{\prime}} \mathrm{d} \rho^{\prime \prime} \rho^{\prime \prime} f\left(\rho^{\prime \prime}\right) .
$$

Here, the point indicated by the normalized co-ordinates $\left(\rho_{P H},-\zeta_{0}\right)$ is linked by a magnetic field line to the point $\left(\rho_{P}, \zeta_{0}\right)$.

For $r<r_{P H}$ and $r_{0}<r_{P}, n\left(r,-z_{0}\right) / n\left(r_{0}, z_{0}\right)=f\left(r / \lambda_{D}\right)$, and the Boltzmann density relation gives $f(\rho)=\exp \left[\psi\left(\rho_{0}, \zeta_{0}\right)-\psi\left(\rho,-\zeta_{0}\right)\right]$. Expanding the exponential and keeping only the lowest order term yield

$$
f(\rho)-1=\psi\left(\rho_{0}, \zeta_{0}\right)-\psi\left(\rho,-\zeta_{0}\right) .
$$

Equations (8), (10), and (11) are combined to write the integral equation

$$
\begin{aligned}
f(\rho)-1= & \frac{\rho_{P}^{2}}{2}\left[\ln \left(\frac{\rho_{W}}{\rho_{P}}\right)+\frac{1}{2}\right]-\frac{\rho_{0}^{2}}{4}-\ln \left(\frac{\rho_{W}}{\rho_{P H}}\right) \\
& \times \int_{0}^{\rho_{P H}} \mathrm{~d} \rho \rho f(\rho)-\int_{\rho}^{\rho_{P H}} \frac{\mathrm{d} \rho^{\prime}}{\rho^{\prime}} \int_{0}^{\rho^{\prime}} \mathrm{d} \rho^{\prime \prime} \rho^{\prime \prime} f\left(\rho^{\prime \prime}\right) .
\end{aligned}
$$

The functional dependence, $\rho_{0}\left(\rho,-\zeta_{0}\right)$, was found to be $\rho_{0}=\sqrt{R} \rho$. Similarly, $\rho_{P}=\sqrt{R} \rho_{P H}$, and substitution gives

$$
\begin{aligned}
f(\rho)-1= & \frac{\rho_{P}^{2}}{2}\left[\ln \left(\frac{\rho_{W}}{\rho_{P}}\right)+\frac{1}{2}\right]-\frac{R \rho^{2}}{4}-\ln \left(\frac{\sqrt{R} \rho_{W}}{\rho_{P}}\right) \\
& \times \int_{0}^{\rho_{P} / \sqrt{R}} \mathrm{~d} \rho \rho f(\rho)-\int_{\rho}^{\rho_{P} / \sqrt{R}} \frac{\mathrm{d} \rho^{\prime}}{\rho^{\prime}} \int_{0}^{\rho^{\prime}} \mathrm{d} \rho^{\prime \prime} \rho^{\prime \prime} f\left(\rho^{\prime \prime}\right) .
\end{aligned}
$$

A differential equation for $f(\rho)$ is found by differentiating Eq. (13), multiplying through by $\rho$, and differentiating a second time

$$
\rho \frac{\mathrm{d}^{2}}{\mathrm{~d} \rho^{2}} f(\rho)+\frac{\mathrm{d}}{\mathrm{d} \rho} f(\rho)-\rho f(\rho)+R \rho=0 .
$$

Requiring the solution to have a finite value at $\rho=0$ (along the $z$ axis), the solution is

$$
f(\rho)=R+C I_{0}(\rho) .
$$

Here, $C$ is a constant and $I$ is a modified Bessel function of the first kind. The value of $C$ is determined by evaluating Eq. (13) at $\rho=0$

$$
\begin{aligned}
R+C I_{0}(0)-1= & \frac{\rho_{P}^{2}}{2}\left[\ln \left(\frac{\rho_{W}}{\rho_{P}}\right)+\frac{1}{2}\right]-\ln \left(\frac{\sqrt{R} \rho_{W}}{\rho_{P}}\right) \\
& \times \int_{0}^{\rho_{P} / \sqrt{R}} \mathrm{~d} \rho \rho\left[R+C I_{0}(\rho)\right] \\
& -\int_{0}^{\rho_{P} / \sqrt{R}} \frac{\mathrm{d} \rho^{\prime}}{\rho^{\prime}} \int_{0}^{\rho^{\prime}} \mathrm{d} \rho^{\prime \prime} \rho^{\prime \prime}\left[R+C I_{0}\left(\rho^{\prime \prime}\right)\right] .
\end{aligned}
$$

Carrying out the integrals and algebraic manipulation gives

$$
\begin{aligned}
C+R-1= & -\frac{\rho_{P}^{2}}{2} \ln (\sqrt{R})+C-C I_{0}\left(\frac{\rho_{P}}{\sqrt{R}}\right) \\
& -C \frac{\rho_{P}}{\sqrt{R}} \ln \left(\frac{\sqrt{R} \rho_{W}}{\rho_{P}}\right) I_{1}\left(\frac{\rho_{P}}{\sqrt{R}}\right),
\end{aligned}
$$

which is linear with respect to $C$ and is solved to find

$$
C=\frac{1-R-\frac{\rho_{P}^{2}}{2} \ln (\sqrt{R})}{\frac{\rho_{P}}{\sqrt{R}} \ln \left(\frac{\sqrt{R} \rho_{W}}{\rho_{P}}\right) I_{1}\left(\frac{\rho_{P}}{\sqrt{R}}\right)+I_{0}\left(\frac{\rho_{P}}{\sqrt{R}}\right)} .
$$

The expression obtained is a function $f(\rho)$, which gives the density at position $\left(r,-z_{0}\right)$ in the high field region when multiplied by the density at the position $\left(r_{0}, z_{0}\right)$ in the low field region, i.e., $n\left(r,-z_{0}\right)$ $=f\left(r / \lambda_{D}\right) n\left(r_{0}, z_{0}\right)$. A comparison of the analytical radial density profile in the high field region and the computed radial density profile for the base case is shown in Fig. 4. Furthermore, the Boltzmann density

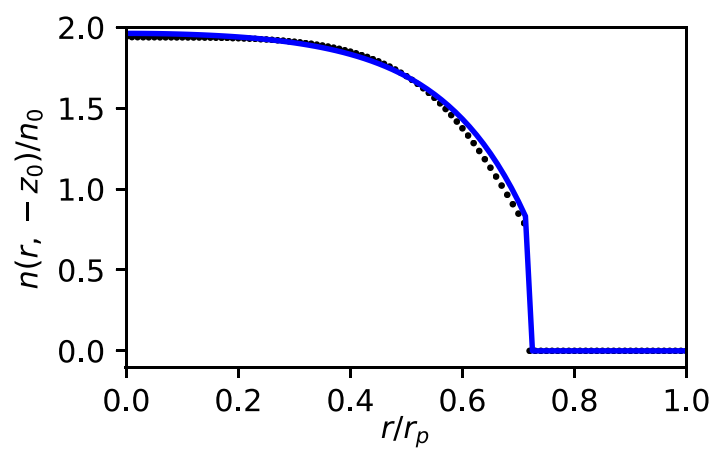

FIG. 4. A comparison of the radial density profile in the high field region calculated analytically and the profile obtained through relaxation methods, for the base case. 
distribution yields that in normalized co-ordinates $\ln f(0)$ $=e\left[\phi\left(0, z_{0}\right)-\phi\left(0,-z_{0}\right)\right] /\left(k_{B} T\right)=e \Delta \phi /\left(k_{B} T\right)=\Delta \psi$. The normalized axial potential difference is

$$
\Delta \psi=\ln \left[R+\frac{1-R-\frac{\rho_{P}^{2}}{2} \ln (\sqrt{R})}{\frac{\rho_{P}}{\sqrt{R}} \ln \left(\frac{\sqrt{R} \rho_{W}}{\rho_{P}}\right) I_{1}\left(\frac{\rho_{P}}{\sqrt{R}}\right)+I_{0}\left(\frac{\rho_{P}}{\sqrt{R}}\right)}\right],
$$

where $I_{0}(0)=1$ has been used. The values for the axial potential difference calculated using Eq. (18) are within 5\% of the axial potential differences in Table I for the computed equilibria, except for the equilibrium with $R=11$ where it is within $15 \%$. The density ratio along the axis is

$$
\frac{n\left(0,-z_{0}\right)}{n_{0}}=e^{\Delta \psi}
$$

In Fig. 5, the predicted and computed density variations along the $z$ axis are compared for equilibria with various Debye lengths.

\section{TWO AND THREE-SPECIES PLASMAS}

The electric potential difference associated with a change in magnetic field strength may be capable of axially confining additional charged species. The additional species may be confined to either a high or low potential region depending on the sign of charge. The potential difference may be used to effectively separate two of the components of a three-species plasma.

The possible formation of a three-species equilibrium where two oppositely charged species are kept in isolation from each other may have applications in antihydrogen research. The ALPHA and ATRAP experiments confine antihydrogen with a magnetic minimum field. The magnetic minimum is formed from the superposition of a transverse multipole field and axial mirror fields on a Malmberg-Penning trap's uniform field. Charged particles are confined as in a MalmbergPenning trap, while antihydrogen atoms are confined through the interaction of their intrinsic magnetic moment with the magnetic minimum field. Only sufficiently cold antihydrogen in the low field seeking state is confined. ${ }^{11}$ Comparisons between antihydrogen and hydrogen can provide fundamental tests of CPT (charge conjugation,

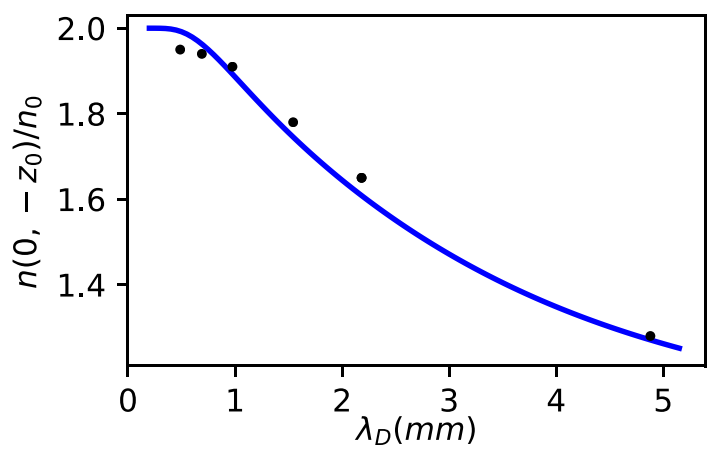

FIG. 5. The density variation along the $z$ axis from the computed equilibria are compared to the density variation predicted analytically (solid line) for equilibria with different Debye lengths. Equilibria in Table I with a parameter varied other than plasma density $n_{0}$ or plasma temperature $T$ are excluded. parity, and time reversal) and/or gravitational symmetries. ${ }^{12}$ Such tests are the goal of multiple collaborations including ALPHA, ${ }^{13}$ ATRAP, ${ }^{14}$ ASACUSA, ${ }^{15,16}$ AEGIS, ${ }^{17,18}$ and GBAR. ${ }^{19}$ The ALPHA and ATRAP collaborations conduct experiments on trapped antihydrogen and have demonstrated production of antihydrogen and confinement for time scales of about $1000 \mathrm{~s} .{ }^{14,20}$

A previous study predicted that space charge based antiproton confinement is possible within the ALPHA experimental apparatus. ${ }^{8}$ A positron plasma would extend axially beyond two coaxial magnetic mirrors, one of which is considered hereafter to be centered at cylindrical co-ordinates $(r, z)=\left(0,-z_{0}\right)$. The antiprotons would be confined within a three-dimensional electrostatic well, which will be considered hereafter to be centered at $(r, z)=\left(0, z_{0}\right)$. The configuration is considered to be symmetric about the $z=z_{0}$ plane, and only the region $-z_{0} \leq z \leq z_{0}$ is referred to hereafter. In Ref. 8 , it is found that the values for plasma parameters at $(r, z)=\left(0, z_{0}\right)$ may be associated with an antihydrogen-producing three-body recombination rate that occurs faster than other relevant rates in the two-species system. The positron density and temperature would be $n\left(0, z_{0}\right)=n_{0}=2$ $\times 10^{13} \mathrm{~m}^{-3}$ and $T=5 \mathrm{~K}$, respectively. The antiproton density and temperature would be $n_{-}\left(0, z_{0}\right)=n_{0-}=2 \times 10^{11} \mathrm{~m}^{-3}$ and $T_{-}$ $=0.5 \mathrm{~K}$, respectively. Also, the positron plasma density was considered to be radially uniform at $z=z_{0}$ out to a plasma radius of $r_{P}=0.9 \mathrm{~mm}$. The inner wall radius of the trap was $r_{W}=22.3 \mathrm{~mm}$, the mirror ratio was $R=2.0$, and an axial potential well depth for confining antiprotons was found to be (in temperature units) $e \Delta \phi / k=3.6 \mathrm{~K}$.

The possibility of confining a third species, consisting of lasercooled beryllium ions, is now considered. The ions are singly charged and positive. Two one-dimensional axial electrostatic wells are produced by the positron space charge, with each well centered under a magnetic mirror. Here, beryllium ions are considered to be axially confined within the axial well centered at $z=-z_{0}$. In the present work, the beryllium-ion density and temperature are considered to be $n_{+}\left(0,-z_{0}\right)=n_{0+}=2 \times 10^{11} \mathrm{~m}^{-3}$ and $T_{+}=0.5 \mathrm{~K}$, respectively. The numerical values for the two parameters are the same as those for the antiprotons. For the parameter values chosen, the antiprotons have a negligible effect on the positron equilibrium, and it is assumed that the same is true for the beryllium ions. However, the radial electric field produced by the positron plasma tends to cause centrifugal separation to occur between the beryllium ions and the positrons. Such an effect is considered in Ref. 9 and is not treated here. The change in potential along the $z$ axis between $-z_{0}$ and $z_{0}$ provides an electric field that tends to keep the beryllium ions axially separated from the antiprotons. Figure 6 provides an illustration of the concept by showing a plot of the normalized density of each species along the $z$ axis according to the Boltzmann density relation

$$
\frac{n_{ \pm}(0, z)}{n_{0 \pm}}=\exp \left(\mp \frac{e}{k_{B} T}\left[\phi(0, z)-\phi\left(0, \mp z_{0}\right)\right]\right) .
$$

The laser-cooled beryllium ions would serve to sympathetically cool the positron plasma within the high magnetic field region, while antiprotons are trapped axially separated from the laser-cooled ions within a low field region. For simplicity, a constant electric field is used that produces a change in potential of $e \Delta \phi / k=3.6 \mathrm{~K}$, and Fig. 6 does not present a self-consistently computed equilibrium. A future study using the methods in Refs. 6, 8, and 9 may be able to assess the feasibility of the proposed three-species equilibrium. 


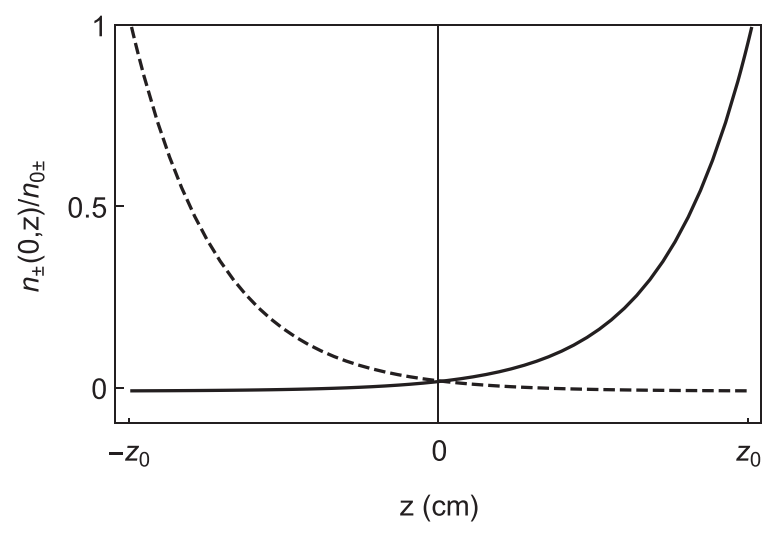

FIG. 6. Illustration of the normalized densities of antiprotons (solid line) and beryllium ions (dashed line) along the $z$ axis. (See text for details.)

\section{DISCUSSION}

The theoretical approach used in this investigation does not simulate time-dependent plasma processes. The time-dependent approach to an equilibrium is not included in the computations, and the effects associated with plasma rotation are neglected. For each equilibrium, iterations are continued until the maximum change in potential between iterations at any grid point is less than $10^{-8} \mathrm{~V}$. The value of $z_{0}$ is chosen such that the magnitude of the finite-length solenoid's magnetic field is less than $0.05 B_{L}$ at $\left(0, z_{0}\right)$. Increasing $z_{0}$ beyond this value was not found to impact the equilibrium potentials obtained by more than $1 \%$.

The techniques developed in this study allow the calculation of the equilibrium of the positron plasma. The plasma is seen to selfconsistently produce a potential difference along the $z$ axis. This potential difference may be useful for antiproton confinement and mixing antiprotons and positrons.

The current work shows correspondence with an analytical study of flat-top, finite-temperature plasmas ${ }^{5}$ and with a particle-in-cell study. ${ }^{6}$ The analysis in Ref. 5 provided four "surprising conclusions." Of the conclusions, three that apply to current plasma equilibria are as follows: the density ratio will scale approximately with the mirror ratio, the electric potential varies along magnetic field lines, and the plasma is "thinner" in the high field region than that would be expected by following field lines. These attributes are seen in the computed equilibria presented here and are in agreement with the analytical description developed in the present work.

In the derivation of the expression for density variation, the plasma in the low field region is assumed to have a uniform charge density out to a plasma radius where it has a sharp edge. The derived expression depends on the accuracy of the assumed density in the low field region. If the density falls off over a distance of the order of the Debye length, the accuracy of the derived expression and the computed equilibria may depend on the Debye length being small compared to the plasma radius. The Debye length is on the order of the plasma radius for some of the equilibria computed.

The methods outlined in Secs. III and V may be generalized to study a variety of configurations with nonuniform magnetic fields. Computations of equilibria with plasmas that do not extend beyond the computational region or that possess nonuniform radial density profiles specified at locations away from the edge of the computational boundary may be feasible.

\section{ACKNOWLEDGMENTS}

Computational resources were provided by UNT's High Performance Computing Services. This material is based upon the work supported by the National Science Foundation under Grant Nos. PHY-1803047 and PHY-1500427 and by the Department of Energy under Grant No. DE-FG02-06ER54883. This endeavor was undertaken at the University of North Texas and does not include any contribution or review by Ball Aerospace.

\section{APPENDIX: MAGNETIC FIELD OF A FINITE-LENGTH SOLENOID}

For a cylindrical finite-length solenoid that is centered at the origin of a cylindrical co-ordinate system, with co-ordinates $(r, \theta, z)$, and that has an axis of symmetry aligned with the $z$ axis, the magnetic field components are (see, for example, Ref. 21)

$$
\begin{aligned}
B_{r}(r, \theta, z)= & \frac{\mu_{0} I n}{\pi} \sqrt{\frac{a}{r m_{+}}}\left(E\left(m_{+}\right)-\left(1-\frac{m_{+}}{2}\right) K\left(m_{+}\right)\right) \\
& -\frac{\mu_{0} I n}{\pi} \sqrt{\frac{a}{r m_{-}}}\left(E\left(m_{-}\right)-\left(1-\frac{m_{-}}{2}\right) K\left(m_{-}\right)\right), \\
B_{z}(r, \theta, z)= & \frac{\mu_{0} I n \zeta_{+}}{4 \pi} \sqrt{\frac{m_{+}}{a r}}\left(K\left(m_{+}\right)+\left(\frac{a-r}{a+r}\right) \Pi\left(u, m_{+}\right)\right) \\
& -\frac{\mu_{0} I n \zeta_{-}}{4 \pi} \sqrt{\frac{m_{-}}{a r}}\left(K\left(m_{-}\right)+\left(\frac{a-r}{a+r}\right) \Pi\left(u, m_{-}\right)\right) .
\end{aligned}
$$

Here, $I$ is the current carried by an infinitesimally thin wire, $I$ is positive or negative in accordance with the right-hand rule, $n$ is the number of wire turns per unit length of the solenoid, $a$ is the radius of the solenoid, $\mu_{0}$ is the permeability of free space (SI units are used), $u=4 a r /(a+r)^{2}, \quad m_{+}=4 a r /\left((a+r)^{2}+\zeta_{+}^{2}\right), m_{-}=4 a r /$ $\left((a+r)^{2}+\zeta_{-}^{2}\right), \zeta_{+}=z+(L / 2), \zeta_{-}=z-(L / 2), L$ is the length of the solenoid, and the complete elliptic integrals of the first, second, and third kinds are

$$
\begin{aligned}
K(m) & =\int_{0}^{\pi / 2}\left(1-m \sin ^{2} \theta\right)^{-1 / 2} d \theta, \\
E(m) & =\int_{0}^{\pi / 2}\left(1-m \sin ^{2} \theta\right)^{1 / 2} d \theta,
\end{aligned}
$$

and

$$
\Pi(u, m)=\int_{0}^{\pi / 2}\left(1-u \sin ^{2} \theta\right)^{-1}\left(1-m \sin ^{2} \theta\right)^{-1 / 2} d \theta .
$$

\section{REFERENCES}

${ }^{1}$ R. L. Spencer, S. N. Rasband, and R. R. Vanfleet, Phys. Fluids B 5, 4267 (1993). ${ }^{2}$ C. A. Ordonez, Phys. Rev. E 67, 046401 (2003).

${ }^{3}$ C. A. Ordonez and D. L. Weathers, Phys. Plasmas 15, 083504 (2008).

${ }^{4}$ J. L. Pacheco, C. A. Ordonez, and D. L. Weathers, Phys. Plasmas 19, 102510 (2012). 
${ }^{5}$ J. Fajans, Phys. Plasmas 10, 1209 (2003); Ref. 9 therein.

${ }^{6}$ K. Gomberoff, J. Fajans, J. Wurtele, A. Friedman, D. P. Grote, R. H. Cohen, and J.-L. Vay, Phys. Plasmas 14, 052107 (2007).

${ }^{7}$ H. Higaki, K. Fukata, K. Ito, H. Okamoto, and K. Gomberoff, Phys. Rev. E 81, 016401 (2010).

${ }^{8}$ R. A. Lane and C. A. Ordonez, J. Phys. B: At., Mol. Opt. Phys. 49, 074008 (2016).

${ }^{9} \mathrm{~N}$. Madsen, F. Robicheaux, and S. Jonsell, New J. Phys. 16, 063046 (2014).

${ }^{10}$ R. M. Hedlof and C. A. Ordonez, AIP Adv. 7, 115123 (2017).

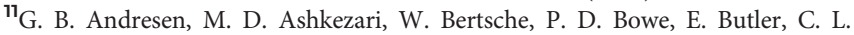
Cesar, S. Chapman, M. Charlton, A. Deller, S. Eriksson, J. Fajans, T. Friesen, M. C. Fujiwara, D. R. Gill, A. Gutierrez, J. S. Hangst, W. N. Hardy, M. E. Hayden, R. S. Hayano, A. J. Humphries, R. Hydomako, S. Jonsell, L. V. Jorgensen, L. Kurchaninov, N. Madsen, S. Menary, P. Nolan, K. Olchanski, A. Olin, A. Povilus, P. Pusa, E. Sarid, S. Seif el Nasr, D. M. Silveira, C. So, J. W. Storey, R. I. Thompson, D. P. van der Werf, and Y. Yamazaki, Nucl. Instrum. Methods Phys. Res., Sect. A 684, 73 (2012).

${ }^{12}$ W. A. Bertsche, E. Butler, M. Charlton, and N. Madsen, J. Phys. B; At., Mol. Opt. Phys. 48, 232001 (2015).

${ }^{13}$ G. B. Andresen, M. D. Ashkezari, M. Baquero-Ruiz, W. Bertsche, P. D. Bowe, E. Butler, C. L. Cesar, S. Chapman, M. Charlton, A. Deller, S. Eriksson, J. Fajans, T. Friesen, M. C. Fujiwara, D. R. Gill, A. Gutierrez, J. S. Hangst, W. N. Hardy, M. E. Hayden, A. J. Humphries, R. Hydomako, M. J. Jenkins, S. Jonsell, L. V. Jorgensen, L. Kurchaninov, N. Madsen, S. Menary, P. Nolan, K. Olchanski, A. Olin, A. Povilus, P. Pusa, F. Robicheaux, E. Sarid, S. Seif el Nasr, D. M. Silveira, C. So, J. W. Storey, R. I. Thompson, D. P. van der Werf, J. S. Wurtele, and Y. Yamazaki, Nature (London) 468, 673 (2010).

${ }^{14}$ G. Gabrielse, R. Kalra, W. S. Kolthammer, R. McConnell, P. Richerme, D. Grzonka, W. Oelert, T. Sefzick, M. Zielinski, D. W. Fitzakerley, M. C. George, E. A. Hessels, C. H. Storry, M. Weel, A. Mullers, and J. Walz, Phys. Rev. Lett. 108, 113002 (2012).

${ }^{15} \mathrm{E}$. Widmann and ASACUSA Collaboration, AIP Conf. Proc. 1441, 546 (2012).

${ }^{16}$ R. Lundmark, C. Malbrunot, Y. Nagata, B. Radics, C. Sauerzopf, and E. Widmann, J. Phys. B: At., Mol. Opt. Phys. 48, 184001 (2015).
${ }^{17}$ D. Krasnicky, S. Aghion, C. Amsler, A. Ariga, T. Ariga, A. S. Belov, G. Bonomi, P. Braunig, R. S. Brusa, J. Bremer, G. Burghart, L. Cabaret, M. Caccia, C. Canali, R. Caravita, F. Castelli, G. Cerchiari, S. Cialdi, D. Comparat, G. Consolati, L. Dassa, S. Di Domizio, L. Di Noto, M. Doser, A. Dudarev, A. Ereditato, R. Ferragut, A. Fontana, P. Genova, M. Giammarchi, A. Gligorova, S. N. Gninenko, S. D. Hogan, S. Haider, E. Jordan, L. V. Jorgensen, T. Kaltenbacher, J. Kawada, A. Kellerbauer, M. Kimura, V. Lagomarsino, S. Mariazzi, V. A. Matveev, F. Merkt, F. Moia, G. Nebbia, P. Nedelec, M. K. Oberthaler, N. Pacifico, V. Petracek, C. Pistillo, F. Prelz, M. Prevedelli, C. Regenfus, C. Riccardi, O. Rohne, A. Rotondi, H. Sandaker, P. Scampoli, J. Storey, M. A. Subieta Vasquez, M. Spacek, G. Testera, R. Vaccarone, F. Villa, and S. Zavatarelli, AIP Conf. Proc. 1521, 144 (2013).

${ }^{18}$ A. Kellerbauer, M. Amoretti, A. S. Belov, G. Bonomi, I. Boscolo, R. S. Brusa, M. Buchner, V. M. Byakov, L. Cabaret, C. Canali, C. Carraro, F. Castelli, S. Cialdi, M. de Combarieu, D. Comparat, G. Consolati, N. Djourelov, M. Doser, G. Drobychev, A. Dupasquier, G. Ferrari, P. Forget, L. Formaro, A. Gervasini, M. G. Giammarchi, S. N. Gninenko, G. Gribakin, S. D. Hogan, M. Jacquey, V. Lagomarsino, G. Manuzio, S. Mariazzi, V. A. Matveev, J. O. Meier, F. Merkt, P. Nedelec, M. K. Oberthaler, P. Pari, M. Prevedelli, F. Quasso, A. Rotondi, D. Sillou, S. V. Stepanov, H. H. Stroke, G. Testera, G. M. Tino, G. Trenec, A. Vairo, J. Vigue, H. Walters, U. Warring, S. Zavatarelli, and D. S. Zvezhinskij, Nucl. Instrum. Methods Phys. Res., Sect. B 266, 351 (2008).

${ }^{19} \mathrm{P}$. Perez and Y. Sacquin, Classical Quantum Gravity 29, 184008 (2012).

${ }^{20}$ G. B. Andresen, M. D. Ashkezari, M. Baquero-Ruiz, W. Bertsche, P. D. Bowe, E. Butler, C. L. Cesar, M. Charlton, A. Deller, S. Eriksson, J. Fajans, T. Friesen, M. C. Fujiwara, D. R. Gill, A. Gutierrez, J. S. Hangst, W. N. Hardy, R. S. Hayano, M. E. Hayden, A. J. Humphries, R. Hydomako, S. Jonsell, S. L. Kemp, L. Kurchaninov, N. Madsen, S. Menary, P. Nolan, K. Olchanski, A. Olin, P. Pusa, C. O. Rasmussen, F. Robicheaux, E. Sarid, D. M. Silveira, C. So, J. W. Storey, R. I. Thompson, D. P. van der Werf, J. S. Wurtele, and Y. Yamazaki, Nat. Phys. 7, 558 (2011).

${ }^{21}$ E. E. Callaghan and S. H. Maslen, "The magnetic field of a finite solenoid," Report No. NASA-TN-D-465, 1960. 